\title{
Methods on scheduling of hydro-photovoltaic complementary system
}

\author{
Huiying Fan ${ }^{1 *}$, Tianxiao Yan $^{1}, \mathrm{Su}_{\mathrm{Guo}}{ }^{1}$ \\ ${ }^{1}$ Hohai University, Nanjing, Jiangsu, 210098, China
}

\begin{abstract}
The hydro-photovoltaic complementarity is an important operation mode to promote the gridconnected absorption, and one of the main directions of solar energy development and utilization in the future. The paper expounds the principle of hydro-photovoltaic complementarity and the influence of the installed ratio on economy, also summarizes and analyzes the research results of hydro-photovoltaic complementary system operation scheduling methods. In view of the uncertainty of hydro-photovoltaic complementary generation, the paper proposes several suggestions and expectations for the study of hydro-photovoltaic complementarity, to provide ideas for improve the economy and reliability of short-term and large-scale hydro-photovoltaic complementary operation.
\end{abstract}

\section{Introduction}

Solar energy resources have the advantages of being abundant, clean, renewable and easy to develop and utilize. photovoltaic power generation technology has the advantages of flexible scale, no pollution and simple maintenance, but at the same time, the output of photovoltaic power generation is affected by geographical and meteorological factors, which lead to some disadvantages such as intermittence and fluctuation. The large-scale directly access to the grid will have many negative effects on the safe and stable operation of the grid $^{[1]}$, this uncontrollability has seriously hindered the development of solar energy, which can be optimized by the quick-start units and energy storage systems of hydropower stations, the adjustability of hydropower makes it be an important link to make multi-energy complementary power generation come true. The key of the hydro-photovoltaic complementarity is how to use the controllable capacity of hydropower station to smooth the photovoltaic generation curve chart, and the energy storage system to shift the peak and fill the valley, according to the output characteristics of photovoltaic generation, so that the photovoltaic generation can be safely connected to the grid, and satisfy the terminally receiving load curve ${ }^{[2]}$.

Multi-energy complementarity is one of the main directions for the development of clean energy in the future, the research on operation and scheduling of hydrophotovoltaic complementary system is of great significance to further promote the high quality of the development of hydro-photovoltaic complementary power generation and improve its economic and environmental benefits. this paper briefly analyzes the theory of hydrophotovoltaic complementary system, and summarizes the research results of hydro-photovoltaic complementary system operation scheduling methods. Then it provides several research ideas for the operation and scheduling of hydro-photovoltaic complementary system and the related work.

\section{Theoretical analysis of hydro- photovoltaic complementarity}

\subsection{Principle of hydro-photovoltaic complementarity}

Hydroelectric power and photovoltaic power have their own advantages and disadvantages. Hydroelectric power has the advantages of being storable and quickly regulated, and the disadvantages of large annual variation. Contemporarily, photovoltaic power has the disadvantages of large seasonal variation, intermittency, instability and non-storability, but its annual variation is very small, and the two also complement each other seasonally. Therefore, the combination of the two can be considered to make up the deficiency and improve the quality. Guaranteeing the original independent power output of the hydropower station, we can adopt the hydrophotovoltaic complementary system, by bundle the photovoltaic power station and the hydropower station into a combined power source, making full use of the quick regulation capacity of the electric generator and the storage function of the reservoir, smoothing the output curve of photovoltaic power generation, shifting the peak to fill the valley, in order to adjust the intermittency and uncertainty of photovoltaic power generation, and improve the quality of photovoltaic power generation, to 
reduce the impact on the power grid, and hydrophotovoltaic complementary system also can improve the utilization ratio of reservoir capacity of hydropower stations, reduce waste water, and take into account economic and environmental benefits.
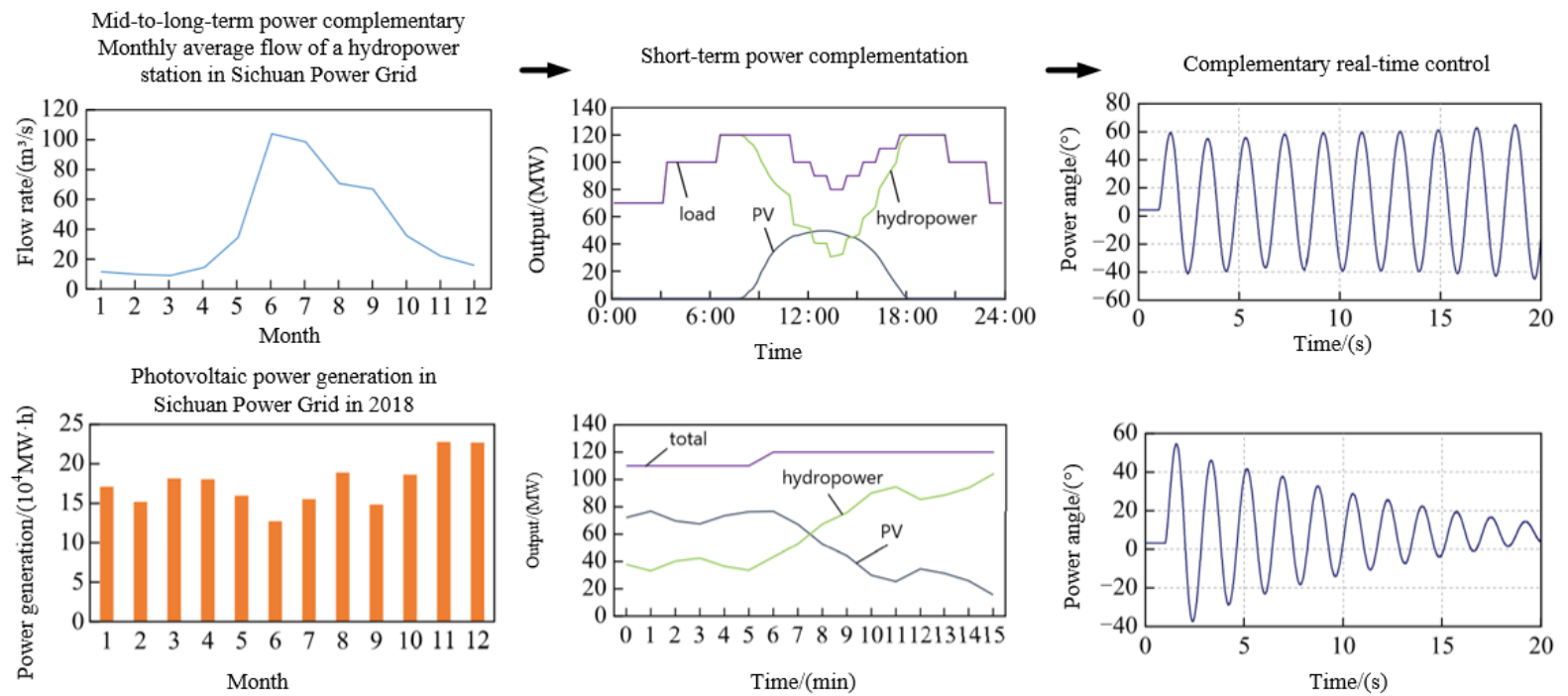

Fig.1 Schematic diagram of hydro-photovoltaic system operating in complementary mode

In the reference [5], the principle of hydrophotovoltaic complementary mode is analyzed from three aspects: long-term power resource complementary, shortterm scheduling complementary and real-time control power fluctuation complementary, as shown in Fig.1.

\subsection{The influence of hydro-solar installed ratio on economy}

Although hydro-photovoltaic complementarity can make the solar energy resources be used effectively, it has a certain adverse effect on the regulation capacity, operation flexibility and overall project economy of hydropower system, but reasonable hydro-solar installed ratio can effectively improve the economic performance of hydrophotovoltaic complementary power supply and give full play to the complementary advantages of hydrophotovoltaic complementarity. In the reference [6], by comparing the simulation and economic calculation of the complementary operation of the Fuxing Dam Hydropower Station and the Dongola Photovoltaic Power Station in Sudan, with the Inga Dams and the Moroccan photovoltaic power station, the economic efficiency of Fuxing Dam Hydropower Station is obviously better than that of Inga Dams Hydropower Station, therefore, several suitable conditions are proposed: (1) The regulation capacity of hydropower station should reach the seasonal regulation and above; (2) When the number of installed hours is less than $4500 \mathrm{~h}$, the equivalent hydro-solar installed ratio of nearly 1:1 can give full play to its advantages, so that it can reduce the integrated landing price, and improve the overall economy and market competitiveness. However, in this reference, there is no good ratio for Inga Dams. It is pointed out in reference [2], which for a hydropower station with daily regulation capacity, and the installed hours are about $6000 \mathrm{~h}$, such as a Inga dams station, the 10:3 installed capacity of the master-slave type can also be made to have better economy, the above-mentioned master-slave type of hydro-solar, names by "hydropowerbased, photovoltaic-assisted" operation mode ${ }^{[7]}$, is the most typical mode in current application, it is also the key of the future research on the hydro-photovoltaic complementary system, but the mode requires sufficient water storage in front of the dam and large discharge.

\section{Research methods of hydro- photovoltaic complementary scheduling}

Renewable Energy Systems, such as wind power and photovoltaic power, have great uncertainty, which brings great difficulties to the scheduling. To solve this problem, many researchers have done a lot of research, using various objective functions to achieve multi-objective optimization, such as flow scheduling, reservoir operating rules modification, reservoir design parameters tuning, and cost optimization etc. All algorithms performed well in this regard particularly multiple objective function design. Reference [9] divides the methods into three levels, to deal with renewable energy uncertainty: prediction, advance planning and real-time scheduling. The prediction of the output of renewable energy is the prerequisite for its large-scale access, and then, taking into account the prediction value, prediction accuracy and operation economy of renewable energy, made a plan in advance to make the power system run at a suitable operation point, finally, the node voltage and branch power flow, caused by the uncertainty of renewable energy, are eliminated by real-time scheduling, such as the reduction of photovoltaic output and the regulation of quick start-up units.

Reference [10] focuses on urban distributed hydrophotovoltaic complementary system, considering the change of power system marginal cost under load change, in order to minimize the cost. Reference [11], considering the penalty cost and reserve cost caused by the underestimation and overestimation of the predicted 
power of renewable energy, through the multi-objective environmental-economic study of wind power and run-ofriver small hydropower, establishes a bi-objective scheduling model considering both fuel cost and pollution emission, and proposes an improved multi-objective optimization algorithm for non-dominated scheduling, the simulation results show that the scheduling model is reasonable and the algorithm is effective. However, this paper focuses more on the study of wind-water complementarity, but less on the interaction of water and solar on different spatial and temporal scales, at the same time, this paper aims at the lowest cost of power generation and the lowest emission of pollution, and put more attention to the cost estimation, but the quality requirements of hydro-photovoltaic complementary power generation and fluctuation rate are neglected.

Reference [1] puts forward the concept of "virtual hydropower", which means that the photovoltaic power station is treated as a non-adjustable unit in a hydropower plant. In Reference [3], a multi-objective scheduling model is established to maximize the output and minimize the fluctuation of the photovoltaic power plant, which takes into account the uncertainty of the photovoltaic output and the resulting forecast bias, the range of prediction is reduced and the strategy of staged fluctuation control is adopted to minimize the fluctuation, but the number of stages should not be too many, having fully considered the constraints of water balance in and between hydropower stations, the constraints of water storage and discharge of reservoirs, and the constraints of power output of hydropower stations, finally, through the case analysis of cascade hydropower stations in Xiaojinchuan River basin and the photovoltaic power stations around it, the conclusion is drawn that the staged fluctuation control strategy is more suitable for the hydro-photovoltaic complementary scheduling than the whole fluctuation control strategy, when the photovoltaic scale is large or the hydropower regulation ability is small. However, the method does not consider many factors when defining rich light and deficient light in different stages, besides, it is not suitable for cloudy and rainy weather, and the time lag of water flow can't be taken into account in solving the model, it is necessary to perfect the forecasting method of hydro-photovoltaic complementary output to improve the scheduling model.
In reference [12], taking into account the influence of different scenarios on hydro-photovoltaic complementarity, according to the three typical weather conditions of sunny, cloudy and rainy combined with two typical water supply in flood season and non-flood season, six calculation schemes are designed and analyzed, and a short-term optimal operation model based on daily operation period is established, which fully considers the water balance, water level, outflow and output constraints of the reservoir, as well as the power balance, peak value correspondence and total output variable amplitude constraints of the power system, the improved step-bystep Optimization Algorithm ${ }^{[9]}$ is used to solve the model. Through the analysis of the model optimization results under six typical scenarios, it can be concluded that, as photovoltaic generation and hydropower are complementary in season, the effect of hydro-photovoltaic complementarity is ideal as long as the reservoir water level does not reach the limited water level in flood season, no matter what the water supply is, there is no abandoned water. On the other hand, abandoned solar in non-flood season is generally more than flood season, and sunny peak period more than rainy weather. The reference only considers the effect of scenarios on abandoned solar and water, but not the fluctuation of output.

Therefore, the uncertainty modeling of hydrophotovoltaic complementary output and load variation is very important to the operation and planning of power grid, scenarios analysis is the key to solve this problem. Reference [13] proposes a scenario analysis technique, which includes the classification of typical scenarios, the analysis of water inflow and photovoltaic generation under each scenario and combination of the scenario. In reference [14], a depth-embedding clustering method is proposed to generate the uncertainty source scenarios of water optical charge. The method uses stack self-coding network to extract the initial characteristics of the uncertainty variable of water optical charge, by iterative optimization of the embedded feature vectors, the timespace dependence of the uncertainty variables of hydropower/PV/load is obtained, and many scenarios are generated, as shown in Fig.2, this method simulates the uncertainty of hydropower/PV/load in several scenarios, which is more accurate than six scenarios. 


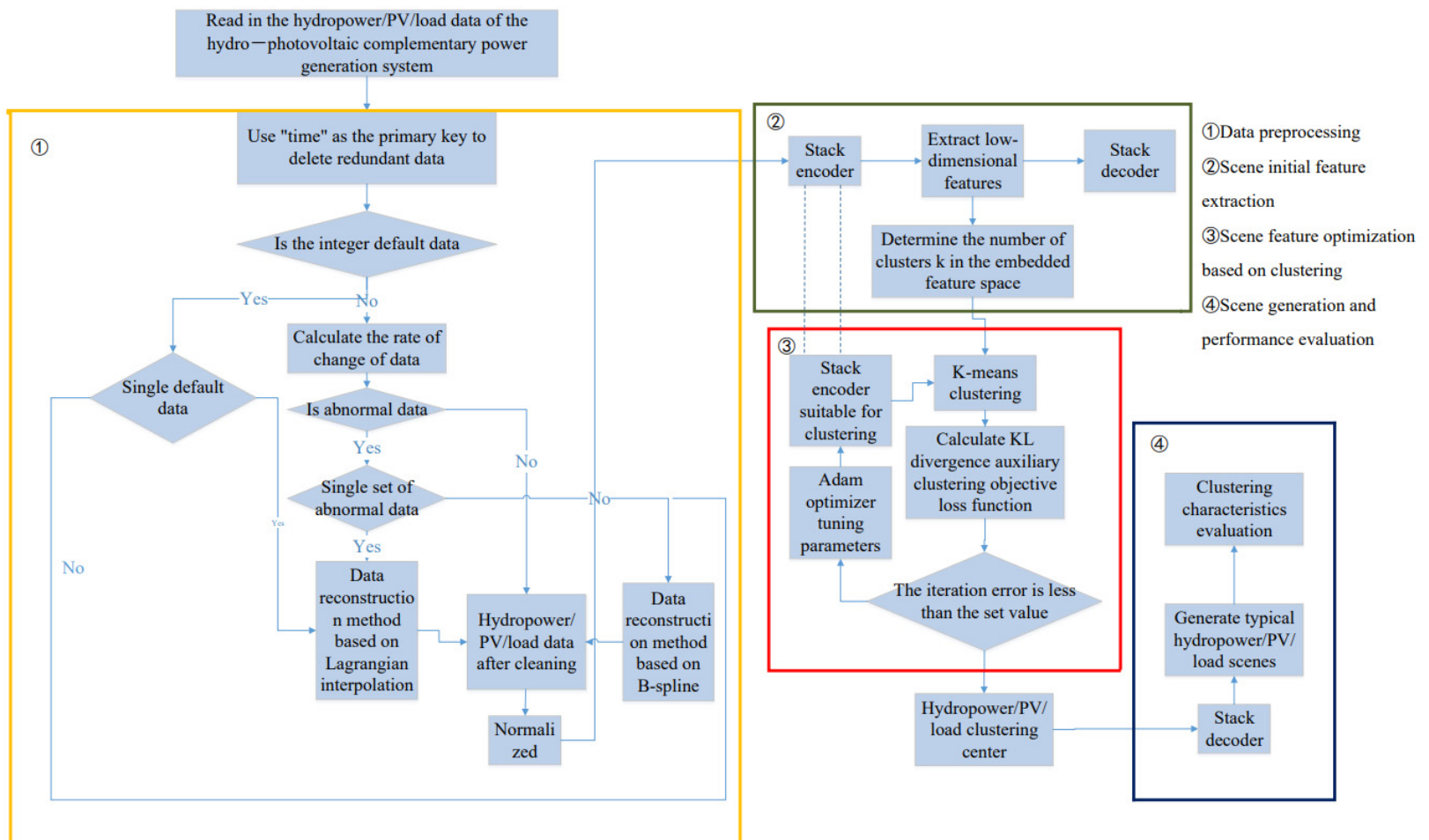

Fig. 2 Framework structure of hydropower/PV/load scenarios generation based on DEC

Reference [15] considers that the factor analysis method is suitable for the classification of renewable energy complementary systems, thus proposes a power classification method for different energy sources, which is especially suitable for large areas, and proposes a multivariable renewable energy complementary output scheduling optimization model, which is helpful to the modeling of multi-energy complementary operation and scheduling.

In conclusion, for the uncertainty of photovoltaic generation, the depth-embedding clustering method can be used to generate a number of scenarios, which can be used to forecast and plan in advance in different scenarios, in order to minimize the fluctuation, a staged fluctuation control strategy can be used for real-time scheduling, and the price caused by penalty cost and reserve cost can be considered.

\section{Conclusions}

Firstly, this paper discusses the principle of hydrophotovoltaic complementarity and the influence of its installed ratio on economy, summarizes and analyzes the researches on how to deal with the uncertainty of photovoltaic power generation. In order to obtain an economical and high-robust scheduling method for hydrophotovoltaic complementary operation, in this paper, the following suggestions are put forward:

1)Selecting the appropriate hydro-solar installed ratio, according to the regulating capacity of hydropower station and the number of the installed hours.

2)The penalty cost and reserve cost, caused by the forecast error of photovoltaic output, can be added to the generation cost to further minimize the cost.
3)The method of scenarios generation can effectively optimize the scheduling and reduce the adverse effects, caused by the uncertainty of photovoltaic generation.

4)Short-term staged fluctuation control strategy for real-time scheduling can effectively smooth the output curve, and it is also easy to dispatch other power sources in time to ensure the total output.

\section{Acknowledgements}

This paper is one of the periodic results of " Forecast of Hydro-photovoltaic Output in Hydro-photovoltaic Complementary System Research", an innovation and entrepreneurship training program for college students in Hohai University. Thanks to Hohai University for providing financial support.

\section{References}

1. Kang B (2020) Summarization of Research on Coordination Operation of Hydropower and PV power in Longyangxia Hydropower Station. Northwest Hydro, 2020(01):23-26.

2. Deng Z, Xie Y, Xiao J, Yang Y (2019) Economic Research and Case Analyses of Large-scale HydroPhotovoltaic Hybrid Power Generation Project Including Long-distance Power Transmission. Hydroelectricity, 2019,45(12):105-108+122.

3. Zhu Y, Chen S, Ma G, Han X, Wang L (2020) ShortTerm Complementary Operation of HydroPhotovoltaic Integrated System Considering Power Generation and Output Fluctuation. Journal of Electrical Engineering Technology, 2020,35(13):2769-2779. 
4. Pang X, Zhang W (2017) Research and application of large-scale water-light complementary coordinated operation technology. Qinghai Science and technology, ,2017,24(02):24-28.

5. Han X, Ding L, Chen G, Liu J, Lin J (2020) Key Technologies and Research Prospects for Cascaded Hydro Photovoltaic-Pumped Storage Hybrid Power Generation System. Journal of Electrical Engineering Technology, 2020,35(13):2711-2722.

6. Deng Z, Yang Y, Wen B (2019) Cast study on the economic feasibility of large-scale hydro-solar power complementary in Africa. Sichuan building materials, 2019,45(11):214-215.

7. Liu W (2020) Current Situation and Countermeasures of Photovoltaic Power and Hydropower Complementary Generation in Xizang. Hydropower and new energy, 2020,34(01):68-71.

8. W. Yuan et al, "Photovoltaic capacity optimization of small and medium-sized hydro-photovoltaic hybrid energy systems considering multiple uncertainties," Journal of Cleaner Production, vol. 276, pp. 124170, 2020.

9. Guo C, Huang Y, Wang L, Pan Y, Dai S (2014) Overview for collaborative dispatch of stochastic power supply and conventional power supply. Journal of Electric Power Science and technology, 2014,29(04):5-11.

10. Jiang L, Xu M, Zhou Y (2020) Optimal configuration of hydro-solar complementary distributed power systems in cities. Huadian Technology, 2020,42(02):58-62+75.

11. Zhang J, Xiong G, Zou X, Yuan X (2021) Multi objective Economic-Environmental Dispatch Considering Wind Power and Small Runoff Hydropower. Power System Automation, 1-11[202103-02].

12. Jia Y, Lin M, Dong Z (2020) Research on Optimal Operation of Hydro Photovoltaic Complementarity in Longyangxia Hydropower Station. Hydroelectric Energy Science,2020,38(10):207-210+106.

13. A.S. Azad et al, "Optimization of the hydropower energy generation using Meta-Heuristic approaches: A review," Energy Reports, vol. 6, pp. 2230-2248, 2020.

14. Yang J, Liu J, Han X, Liu J, Ding L, Zhang S, Hu S (2020) An Uncertain Hydro/PV/Load Typical Scenarios Generation Method Based on Deep Embedding for Clustering. Chinese Journal of Electrical Engineering, 2020,40(22):7296-7306.

15. de Oliveira Costa Souza Rosa, Caroline et al, "Assessing complementarity and optimizing the combination of intermittent renewable energy sources using ground measurements," Journal of Cleaner Production, vol. 258, pp. 120946, 2020. 